\title{
Acute Digital Ischemia Associated with Closed Injury
}

\author{
David Janák ${ }^{1,4}$, David Ručka², Jaroslav Kudlička ${ }^{3}$, Vilém Rohn', \\ Jaroslav Lindner ${ }^{1}$, Otomar Kittnar ${ }^{4}$
}

${ }^{1} 2^{\text {nd }}$ Department of Surgery - Department of Cardiovascular Surgery, First Faculty of Medicine, Charles University in Prague and General University Hospital in Prague, Prague, Czech Republic;

${ }^{2} 2^{\text {nd }}$ Department of Medicine - Department of Cardiovascular Medicine, First Faculty of Medicine, Charles University in Prague and General University Hospital in Prague, Prague, Czech Republic;

${ }^{3} 3^{\text {rd }}$ Department of Medicine - Department of Endocrinology and Metabolism, First Faculty of Medicine, Charles University in Prague and General University Hospital in Prague, Prague, Czech Republic;

${ }^{4}$ Institute of Physiology, First Faculty of Medicine, Charles University in Prague, Prague, Czech Republic

Received May 11, 2015; Accepted September 15, 2015.

Key words: Ischemia - Trombolysis - Closed injury

Abstract: Injury of an artery has a significantly worse prognosis for the patient than a venous injury. Blunt injuries of lower limb digital arteries with the development of acute ischemia present a very rare phenomenon. A crush mechanism with a defect of the non-wetted surface of vessel's inner part and the development of subsequent thromboischemic lesion is essential for the development of ischemia. We report a blunt injury of the right lower limb in a patient after incorrect stepping with subsequent lesion of digital arteries and the development of acute acral ischemia of the right toes.

Mailing Address: David Janák, MD., $2^{\text {nd }}$ Department of Surgery - Department of Cardiovascular Surgery, First Faculty of Medicine, Charles University in Prague and General University Hospital in Prague, U Nemocnice 2, 12800 Prague 2, Czech Republic; e-mail: janakdavid@seznam.cz 


\section{Introduction}

An overview of the relevant literature regarding the issue of closed injury of digital arteries is not extensive. The world literature includes a small quantity of case reports, and one retrospective study (Giddins and Burge, 1996; Reagan et al., 2002). The relevant case reports describe in particular the injury of upper limb digital arteries with compression traumas affecting fingers (Faraj and Craigen, 1998). The most common pattern involves a compression finger trauma with a transverse phalangeal fracture in proximity to the interphalangeal joints and avulsion, or with the distension of digital arteries, with the consequential hypo-perfusion of a finger and the development of acute ischemia. Classical diagnostic symptomatology in patients involves reduced sensitivity, cyanosis or paleness, painful fingers, capillary emptiness with the development of acute ischemia. The involvement of digital arteries of lower limbs is very rare; in our case without any joint dislocation and any skeletal fracture, and after a minimum trauma.

\section{Case report}

A 43-year-old, smoker, after a femur fracture in 2008 on the right, was admitted to our centre due to acute symptomatic ischemia Ilb according to the Rutherford classification of the right lower limb with acral manifestation. Problems appeared suddenly, when he was getting off a train and stepped from the elevated position. The patient came 6 hours after the event with a painful metatarsus and violet toes. The patient was examined additionally, an X-ray examination of the lower limb, without any evidence of bone trauma. Sonographic examination of popliteal artery excluded the syndrome of entrapment. The angiography of lower limb was carried out. The angiography confirmed incomplete convexity of the plantar arch and the obliteration of digital arteries (Figure 1A). Local thrombolysis with a recombinant tissue plasminogen activator (t-PA) in the dose of $0.9 \mathrm{mg} / \mathrm{kg}$ together with 5000 heparin units, then 500 heparin units/hour, in an arterial infusion with partial recanalization was carried out with suboptimal outcome. We managed to unblock the digital arteries into the area of proximal phalanges. Distal obstruction continued (Figure 1B). An intravenous vasodilatation therapy with Prostavasin combined with a hyperbaric chamber for 90 min during 5 days at max. 2.5 ATA (absolute atmosphere) was launched. During hospitalisation, analgesic therapy was administered to the patient repeatedly with intravenous and per os nonsteroidal anti-inflammatory drugs (NSAID). Subsequently, after this therapy, a local clinical improvement of the perfusion was observed. Another examination carried out; transesophageal echocardiography excluded a cardial thrombus. The patient was examined by laboratory, which excluded a procoagulant status. (Leiden mutation and mutation in methylenetetrahydrofolate reductase, MTFR, were excluded.) The patient was anticoagulated with warfarin. Despite this therapy, an ischemia occured gradually, including the surface loss of soft tissues mainly of the big toe and of the fourth toe (Figure 2). The patient was discharged early at his own 


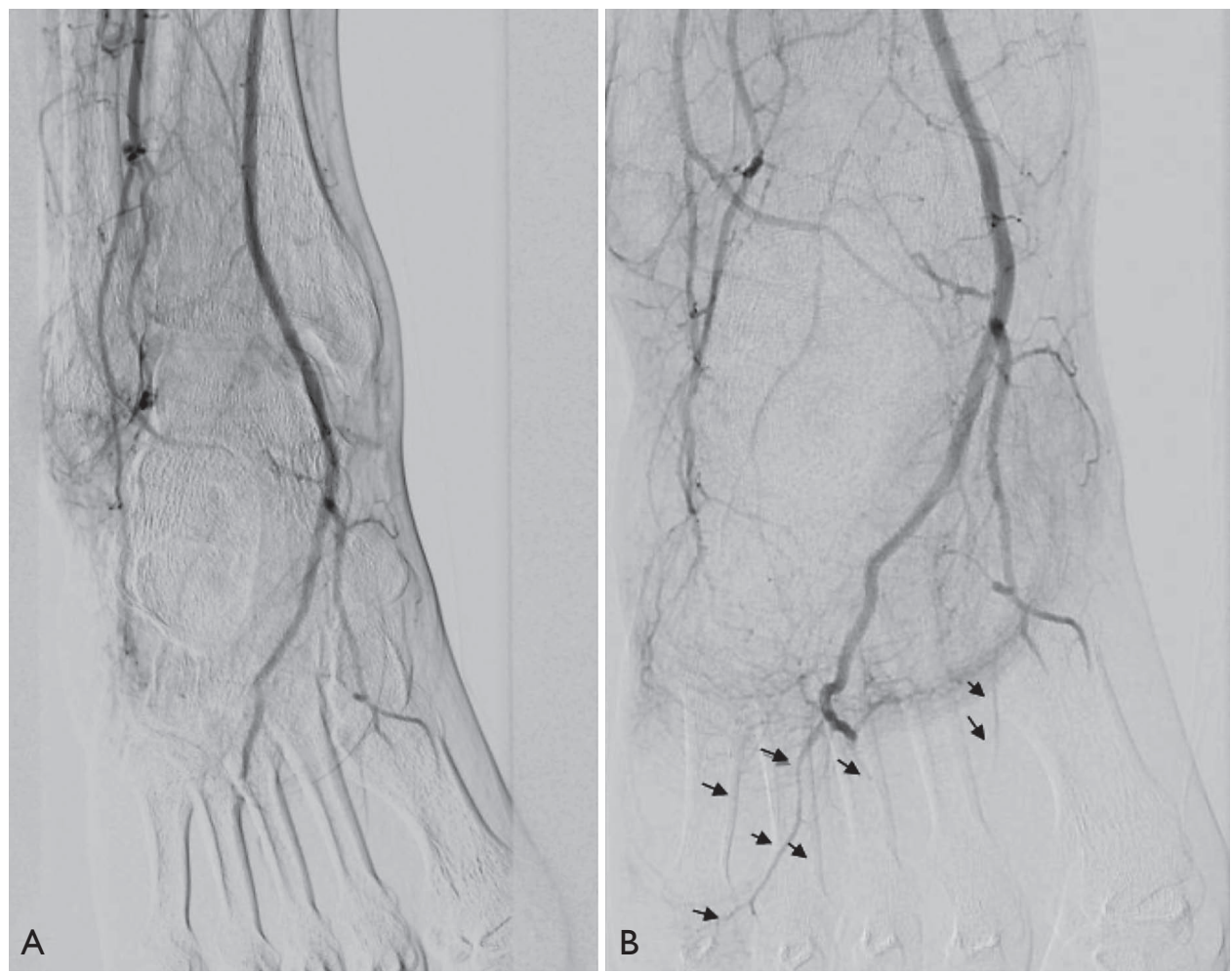

Figure 1 - (A) Primary finding - The obliteration of digital arteries. (B) Partial unblocking (arrows) with residual distal obstruction.

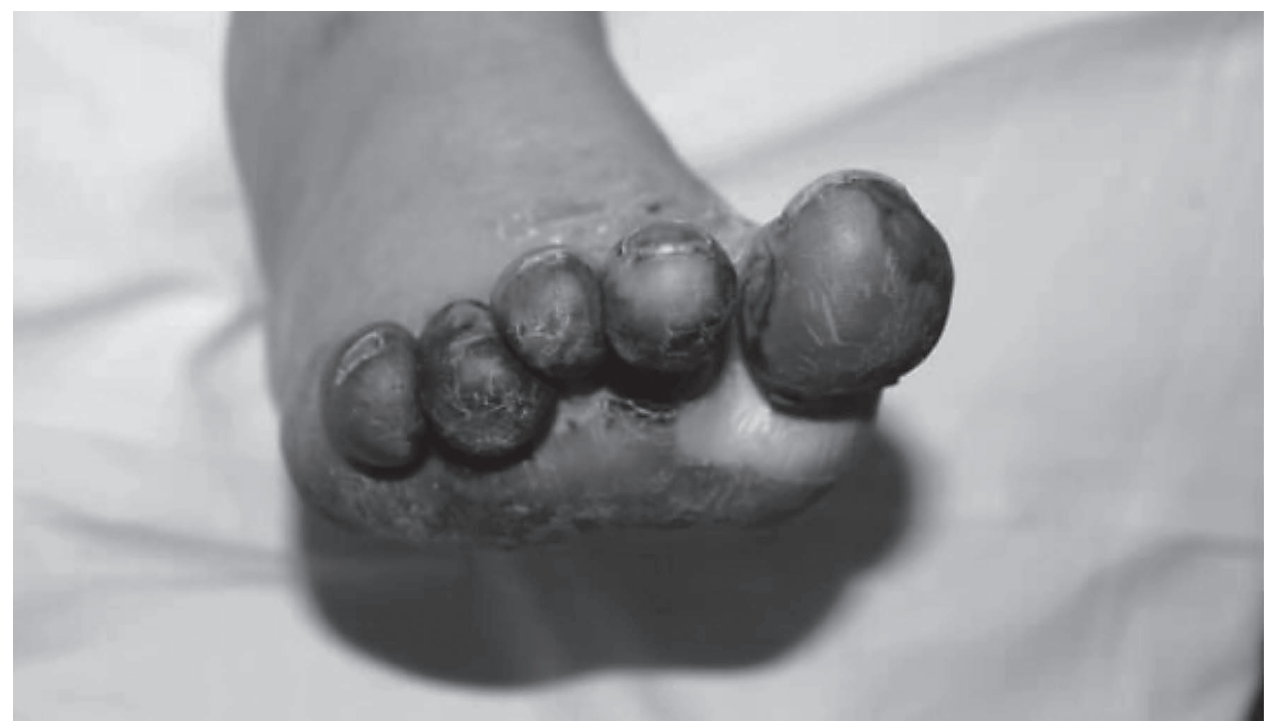

Figure 2 - Clinical stage day 5. 
request for home and outpatient care. Then the patient rejected hospital admission. Warfarinization was recommended for patient's medication. In an examination after more than one year, the patient is after the amputation of the $3^{\text {rd }}, 4^{\text {th }}$ and $5^{\text {th }}$ toes with secondary healing and with a dry defect of the big toe, and with dry necrosis of the $2^{\text {nd }}$ toe.

\section{Discussion}

Arterial injury is a frequent complication of traumas, mainly of multiple traumas (8-10\%) (Hafez et al., 2001; Zellweger et al., 2004). In most cases we encounter direct arterial injuries: Punctured, cutting and blunt arterial injuries with compression and contusion etiology. Indirect arterial injuries of a deceleration and contusion nature are much less frequent. Indirect avulsion injury is mostly associated with joint dislocations. The injury of popliteal artery in knee joint dislocation and the affection of axillary artery in shoulder joint dislocation (McCroskey et al., 1988; Weaver et al., 2000) is the most frequent. In exceptional cases we can experience the deceleration and distension of arteries, and consequently, their thrombosis, depending on the vessel size of such affected arteries. Closed digital artery injuries are identified very rarely in literature. Ultrasound, oximetry and digital subtraction angiography (DSA) present a golden standard for diagnostics. Literature describes successful surgical repairs of affected digital arteries with the restoration of adequate perfusion. Most cases are described for closed compression injury of fingers associated with phalangeal fracture, and avulsions or dissections of digital arteries. Nowadays, with respect to the local finding, endovascular processes are applied very frequently, mainly with the use of targeted thrombolysis. In our case we describe an involvement of digital arteries at the right lower limb after a blunt injury of the sole after incorrect stepping. This specific case involved a total thrombotic occlusion of digital arteries with suspected traumatic distension, dissection and the affection of the non-wetted surface of arteries with subsequent development of arterial occlusion and the ischemia of toes. With respect to the nature of the local finding and the mechanism of the injury, we deduce thrombotic affection of the plantar arteries. We have not found the source of any possible embolization. The patient had a sinus rhythm and in the personal history he was without cardiac arrhythmia. Due to the unfavourable anatomic location and impossibility of an adequate surgical intervention and to the nature of peripheral lesion, we applied local thrombolysis with hyperbaric chamber treatment. We applied the over-pressure treatment to increase the oxidation-reduction potential and the supply of oxygen into the affected tissue to support restorative actions in the affected tissue; with suboptimal result only. This type of injury with an adverse effect on the arterial system with rapid development of acute ischemia poses a great therapeutic challenge, often of uncertain outcome. 


\section{References}

Faraj, A. A., Craigen, M. A. (1998) Closed digital artery injury. J. Accid. Emerg. Med. 15(5), 307.

Giddins, G. E., Burge, P. D. (1996) Closed vascular injury of the finger. J. Bone Joint Surg. Br. 78(2), 325-327.

Hafez, H. M., Woolgar, J., Robbs, J.V. (2001) Lower extremity arterial injury: Results of 550 cases and review of risk factors associated with limb loss. J.Vasc. Surg. 33, 1212-1219.

McCroskey, B. L., Moore, E. E., Pearce, W. H., Moore, F. A., Cota, R., Sawyer, J. D. (1988) Traumatic injuries of the brachial artery. Am. J. Surg. 156, 553-555.

Reagan, D. S., Grundberg, A. B., Reagan, J. M. (2002) Digital artery damage associated with closed crush injuries. J. Hand Surg. Br. 27(4), 374-377.

Weaver, F., Hood, D. B., Yellin, A. (2000) Vascular injuries of the extremities. In: Vascular Surgery, $5^{\text {th }}$ Edition. Rutherford, R. B., pp. 862-871, W.B. Saunders, Philadelphia.

Zellweger, R., Hess, F., Nicol, A., Omoshoro-Jones, J., Kahn, D., Navsaria, P. (2004) An analysis of 124 surgically managed brachial artery injuries. Am. J. Surg. 188, 240-245. 